\title{
Study on Adaptive Channel Estimation in OFDM Systems Based on DSP/FPGA
}

\author{
L. Ye, Zhang You chun* \\ Engineering and Electronic Information Department \\ Wuhan University of Engineering Science \\ Wuhan, Hubei, China
}

\begin{abstract}
In order to solve channel estimation problem in OFDM (orthogonal frequency division multiplexing) systems, a decision-directed method is proposed for channel estimation and equalization based on OFDM. The proposed method uses a received subcarrier-symbol to represent the competitive neural network, uses a nerve cell of the neural network to estimate adaptively the variation weight value, and then estimates the channel according to the channel weight value. Finally the channel equalization is reached by removing the constellation symmetry to compensate the nonlinearity effect. This method does not require the prearrangement knowledge about the wireless communication channel. Simulations and actual test show that the proposed method achieves very good channel estimation and performance equalization.
\end{abstract}

Keywords-OFDM systems; direct decision estimation; nerve-cell network; channel estimation

\section{INTRODUCTION}

As a very promising technology, OFDM (orthogonal frequency division multiplexing) communication has made great progress. Currently, OFDM systems have been widespreadly used in high-speed data transmission. The coherent detection technology is used for frequency selection and time-varying channel estimation, which requires the accurate channel impulse response information. And the channel impulse response is also used for channel estimation, which usually requires pre-set pilot symbols, but to some extent it reduces the transmission rate of the data [1]. The literature [2] proposed a pilot optimization method for time-varying, slow fading channels, as well as time- varying, frequency-selective channels. The literatures [3-5] also proposed a blind channel estimation and channel equalization method for OFDM systems with high data rate and fast fading channels.

This paper proposed a method for channel estimation in OFDM systems using direct decision-making algorithm, which is a nonlinear algorithm. By independently tracking each subcarrier of the received signal, the method uses the competitive neural network to allocate a neuron for each symbol QPSK (quadrature differential phase shift keying). And it can improve channel estimation performance with redundant information. Also it can improve channel equalization results by eliminating the influence of the constellation symmetrical nonlinear compensation.

The paper is organized as follows. The main algorithm is stated in Section II. Simulation analysis results are presented in Section III. Then the experimental system test is also proposed in Section IV. At last, the conclusions are given in Section V.

\section{SIMULATION ANALYSIS}

The OFDM system is given based on pilot channel estimation in Figure 1. Firstly the binary data is grouped and mapped according to "Map" block. After inserting pilots either to all sub-carriers with a specific period or uniformly between the information data sequence, "IDFT" block is used to transform the data sequence of length $N\{X(k)\}$ into time domain signal $\{X(n)\}$. Following "IDFT" block, guard time is inserted to prevent inter-symbol interference, which is chosen to be larger than the expected delay spread. The transmitted signal $x_{f}(n)$ will pass through the frequency selective time varying fading channel with additive noise. At the receiver, guard time is removed and $y(n)$ is sent to "DFT" block after passing to discrete domain through A/D and low pass filter. Following "DFT" block, the pilot signals are extracted and the estimated channel for the data sub-channels is obtained in "Channel Estimation" block. Finally the binary data is obtained back in "Demap" block.

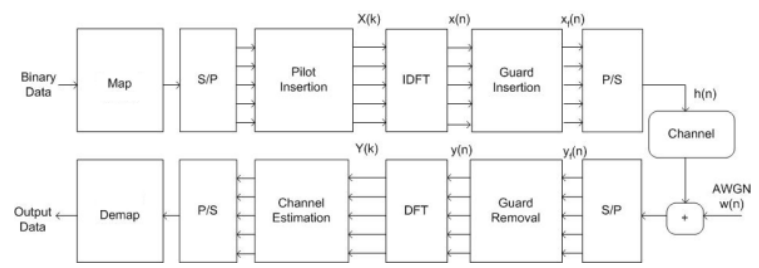

FIGURE I. SYSTEM ARCHITECTURE OF OFDM SYSTEMS.

According to Figure 1, the system structure in MATLAB Simulation is shown in Figure 2. OFDM system parameters used in the simulation are indicated in Table 1. And in the simulation, we have used two multi-path Rayleigh fading channel. We assume to have perfect synchronization since the aim is to observe channel estimation performance. Moreover, we have chosen the guard interval to be greater than the maximum delay spread in order to avoid inter-symbol interference. Simulations are carried out for different signal-to-noise (SNR) ratios and for different Doppler spreads. The channel estimation at pilot frequencies is performed by using direct decision-making algorithm. 
TABLE I. SIMULATION PARAMETERS.

\begin{tabular}{ll}
\hline Parameter & Specifications \\
\hline FFT Size & 1024 \\
Number of Carriers & 128 \\
Pilot Ratio & $1 / 8$ \\
Guard Length & 256 \\
Guard Type & Cyclic Extension \\
Sample rate of OFDM signal & $44.1 \mathrm{kHz}$ \\
Bandwidth & $17.5 \mathrm{kHz}$ \\
Signal Constellation & QPSK \\
\hline
\end{tabular}

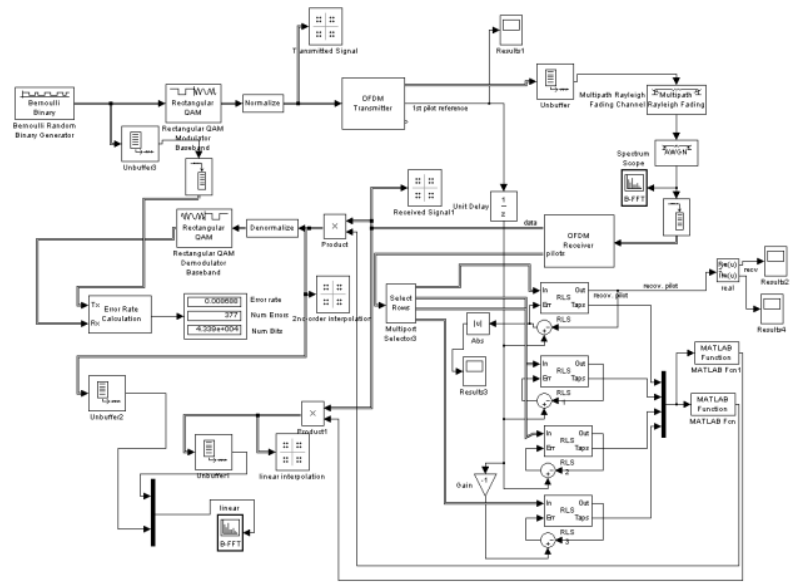

FIGURE II. SYSTEM STRUCTURE IN MATLAB SIMULATION.
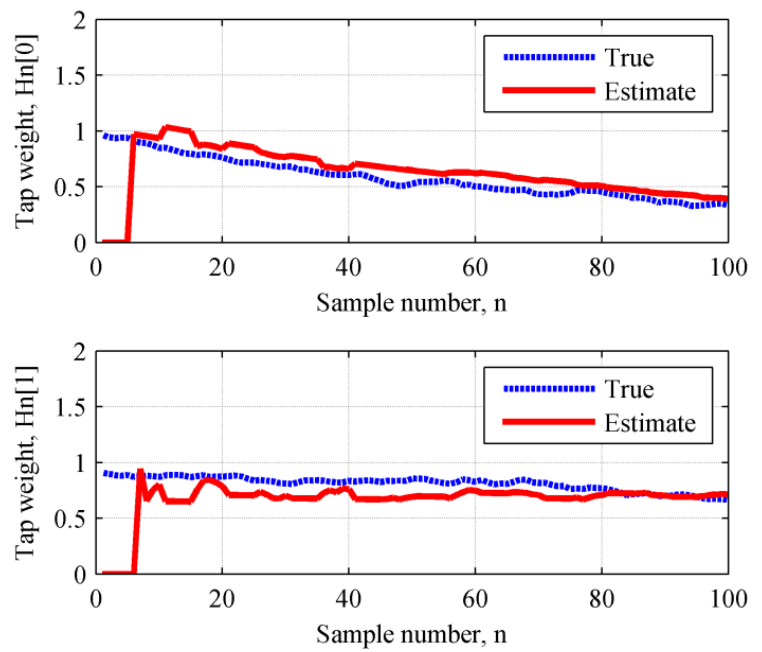

FIGURE III. SIMULATION RESULTS OF CHANNEL ESTIMATION IN OFDM SYSTEMS.

\section{EXPERIMENTAL SYSTEM TEST}

Signal processing data path and control operations make up the bulk of the processing load in a wireless basestation. Most architectures implement the system control, configuration, and the signal-processing data path using a combination of microcontrollers (MCUs), FPGAs, and programmable digital signal processors. The MCU controls the system, while the FPGA and digital signal processor handle the data-flow processing. Systems with light processing demands and control-oriented tasks are realized in software on a digital signal processor; heavier loads are best implemented in FPGAs that provide significant parallel processing benefits. The combination of digital signal processors and FPGAs ensures complete system flexibility and offers reprogrammability to fix bugs or even support entirely different standards.

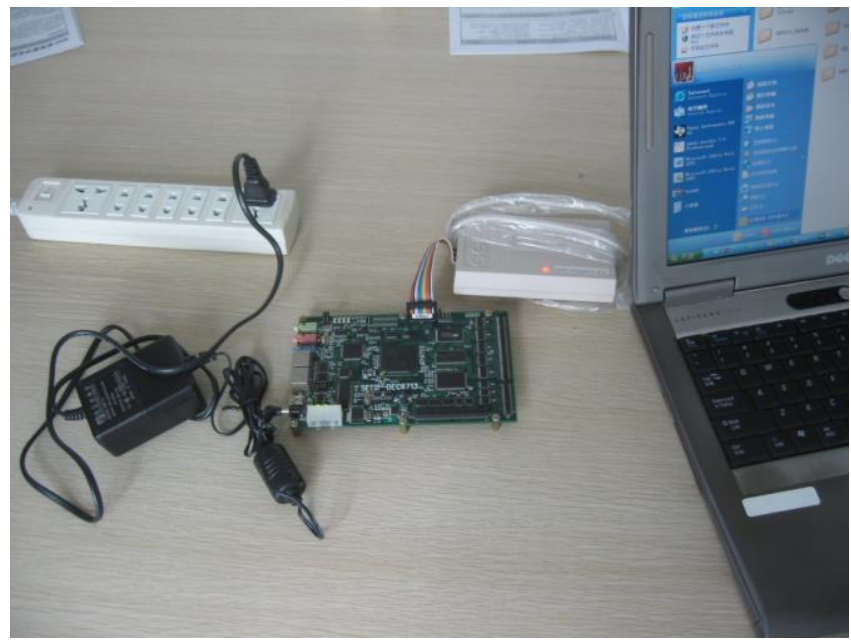

FIGURE IV.SYSTEM BOARD PHOTO.

Here high frequency signal processing unit is mainly to achieve signal amplification, filtering and other functions, analog-to-digital conversion using the A/D chip AD7274 analog signal into a digital signal, the digital signal is then sent to the Xilinx FPGA chip XC2S200PQ208 to complete signal detection, and the modulation, and demodulation of the signal is performed by using DSP. The chip of the modulation and demodulation portion is TI's TMS320C6713, which the codec, error correction, serial communication protocol will be completed in the DSP chip, shown in Figure 4. Here the guard interval takes $25 \mu \mathrm{s}$, OFDM symbol period takes $200 \mu$ s, which is eight times of the interval. The sampling period is $\mathrm{Ts}=200+25=225 \mu \mathrm{s}$, and the frequency band is $400 \mathrm{kHz} \sim 1.5 \mathrm{MHz}$. The bandwidth is divided to 512 sub-carriers, and the sample number takes 750 , then the remaining sub-carriers is $750-512=328$ up to zero. The sampling frequency is 3.75 $\mathrm{MHz}$, and the subcarrier interval is $5 \mathrm{kHz}$.

Experimental tests in the laboratory, the number of bits transmitted is $10 \mathrm{M}$, taking into account the time-varying channel, the tests are carried out in the following different time points, respectively. The distance between transmission data and receive data terminals is about $150 \mathrm{~m}$. Because it is more difficult to detect the SNR of the experimental channel, the effectiveness of the algorithm here by the bit-error rate (BER). The experimental tests show that the average error rate is $5.56 \mathrm{e}-2$ without using channel estimation and equalization. But using channel 
estimation and equalization, channel error rate has decreased, and the average error rate is reduced to $5.27 \mathrm{e}-3$.

\section{CONCLUSIONS}

In this paper, an adaptive method is proposed to channel estimation and equalization in OFDM systems. The method is characterized by significant accurately nonlinear and time-varying channel estimation and tracking of the channel frequency response. In addition, the performance of direct decision-making algorithm is observed by simulation and experimental testing. Results show that the method proposed have better performances in terms of channel estimation and equalization.

\section{REFERENCES}

[1] M. Morelli and U. Mengali, "A comparison of pilot-aided channel estimation methods for ofdm systems," IEEE Trans. on Signal Processing, vol. 49, no. 12, pp. 3065-3073, 2004.

[2] J. W. Choi and Y. H. Lee, "Optimum pilot pattern for channel estimation in ofdm systems," IEEE Trans. on Wireless Communications, vol. 4, no. 5, pp. 2083-2088, 2005.

[3] R. Negi and J. Cioffi, "Pilot tone selection for channel estimation in a mobile ofdm system," IEEE Trans. on Consumer Electronics, vol. 44, no. 3, pp. 1122-1128, 1998.

[4] B. Muquet, M. de Courville and P. Duhamel, "Subspace-based blind and semi-blind channel Estimation for OFDM systems," IEEE Trans. on Signal Processing, vol. 50, no. 7, pp. 1699-1712, 2002.

[5] T. Zemen and C. F. Mecklenbrauker, "Time-variant channel estimation using discrete prolate spheroidal sequences," IEEE Trans. on Signal Processing, vol. 53, no. 9, pp. 3597-3607, 2005.

[6] M. Raugi and M. Tucci, "Power-line communication channel estimation and tracking by a competitive neural network," IEEE Trans. on consumer Electronics, vol. 52, no. 4, pp. 1213-1219, 2006.

[7] S. Zhou and G. B. Giannakis, "Finite-alphabet based channel estimation for OFDM and related multi-carrier systems," IEEE Trans. Communications, vol. 49, no. 8, pp. 1402-1414, 2001.

[8] J. Ran, R. Gruheid and H. Rohling, "Decision-directed channel estimation method for OFDM systems with high velocities," Vehicular Technology Conference, vol.4, pp.22-25, 2003. 